\title{
Why should constant stimulation of saccular afferents modify the posture and gait of patients with bilateral vestibular dys- function? A review.
}

\author{
Ian S. Curthoys ${ }^{1 *}$, Paul F. Smith ${ }^{2}$ and Angel Ramos de Miguel ${ }^{3}$ \\ 1 Vestibular Research Laboratory, School of Psychology, The University of Sydney, Sydney, NSW 2006, Aus- \\ tralia \\ 2 Department of Pharmacology and Toxicology, School of Biomedical Sciences and The Brain Health Re- \\ search Centre, University of Otago, Dunedin, New Zealand \\ 3 Department of Otolaryngology, and Head and Neck Surgery, Complejo Hospitalario Universitario \\ Insular Materno Infantil de Gran Canaria, Las Palmas de Gran Canaria, Spain \\ * Correspondence: ian.curthoys@sydney.edu.au
}

\begin{abstract}
An ongoing EU Horizon 2020 Project called BionicVEST is investigating the effect of constant electrical stimulation (ES) of the inferior vestibular nerve in patients with bilateral vestibular dysfunction (BVD)[1]. The evidence is that constant ES results in improved postural stability and gait performance and so the question of central importance is how could constant ES of mainly saccular afferents in these BVD patients cause this improved performance? We suggest that the constant ES is substituting for the absent saccular neural input to the vestibular nuclei and the cerebellum in these BVD patients and indirectly via these structures to other structures which have been of great recent interest in motor control. One target area, the anterior midline cerebellum (the uvula) has recently been targeted as a location for deep brain stimulation in human patients to improve postural stability and gait. There are projections from midline cerebellum to basal ganglia including the striatum, structures involved in the initiation of gait. It may be that the effect of this activation of peripheral saccular afferent neurons is analogous to the effect of deep brain stimulation (DBS) by electrodes in basal ganglia acting to help alleviate the symptoms of patients with Parkinson's disease.
\end{abstract}

Keywords: Bilateral vestibular dysfunction, saccular, posture, gait, vestibular implant, otolith

\section{Introduction}

Patients with bilateral vestibular dysfunction (BVD) show postural instability and gait difficulties. One treatment for BVD which is under investigation in a multicentre EU project (called BionicVEST) is to implant a long-term stimulating electrode on one inferior vestibular nerve and deliver a constant train of high frequency electrical pulses by that electrode (1200 biphasic pulses per second continuously) and measure the behavioural and perceptual effects of that electrical stimulation (ES). The electrode location is such that the ES is probably mainly stimulating saccular afferents. Patients are selected according to strict criteria to meet the Barany guidelines for BVD. As of 3.1.2022 a total of 10 patients has been implanted.

Preliminary results continue to be obtained, some of which have been published [1]. It is clear that this constant electrical stimulation acts to improve the patient's postural stability and gait performance as measured by Computerized Dynamic Posturography (CDP) and the Dynamic Gait Index (DGI). There are also changes in a number of tests of peripheral vestibular function which are described in detail in [1]. The patients report 
that this constant stimulation is beneficial and do not want it switched off. With this stimulus they find they have less need for assistance in walking and stabilising themselves.

Here the aim is to state a simple hypothesis about why this constant high frequency electrical stimulation could have these effects and to review the evidence relevant to that hypothesis. The hypothesis is called the "saccular substitution hypothesis" - and it is that that the neural activity generated by the ES is substituting for the reduced or absent constant saccular afferent activity from the saccular macula. We review recent evidence relevant to that hypothesis - evidence about the role of direct and indirect vestibular input for postural stability and gait performance.

The saccular stimulation is activating descending spinal pathways as well. It may be that the effect of this saccular stimulation is analogous to the effect of deep brain stimulation (DBS) by electrodes in the basal ganglia acting to help alleviate the symptoms of patients with Parkinson's disease. Possibly the constant stimulation of the saccular afferents in the vestibular nerve is just a step further back: so rather than directly stimulating the basal ganglia electrically, now it is a matter of stimulating saccular afferents electrically which project via the cerebellum to the basal ganglia, and also to the spinal cord and so result in changes in performance.

BionicVEST is a pilot programme to investigate the effect of constant electrical stimulation (ES) of inferior vestibular nerve in patients with BVD [1] according to the consensus criteria of the Barany Society [2]. The aim is to determine if this constant ES results in changes in performance on:

- Tests of peripheral vestibular function,

- Tests of postural stability and gait performance

- Patient subjective experience. Do they find constant ES to be an improvement?

\section{The steps in the research process.}

1. Selection. Patients are selected according to carefully defined selection criteria defining bilateral vestibular dysfunction (BVD). It should be stressed that there is some residual peripheral vestibular function in these patients - but their level of function falls below the international accepted standard for bilateral vestibular dysfunction (Barany Society consensus statement [2])

2. Preoperative testing of peripheral vestibular function, postural stability, and gait (e.g., Using the DGI and CDP etc)

3. The electrode and implantation. A modified cochlear implant electrode is implanted on a branch of the vestibular nerve in one ear. The exact location of the electrode depends on particular anatomical considerations at surgery. The aim is to implant the electrode very close to the inferior vestibular nerve containing afferents from the saccular macula and posterior semicircular canal. From the CT verified electrode locations, it appears that the electrode locations so far have been in the inferior vestibular nerve and so largely otolithic, mainly saccular afferents. It is likely there is some stimulation of the afferents from the posterior canal, however it is necessary to emphasise that this is uncertain. We will refer to the data as being due to saccular stimulation, whereas in fact it more likely should be referred to as vestibular stimulation (probably mainly saccular).

4. Post-operative testing. Initially it is necessary to verify that the stimulating electrode is functional and make adjustments of the stimulus parameters of the ES (relying on patient subjective reports of oscillopsia or subjective sensations.)

5. The stimulus. Then the ES is switched on - a constant train of pulses at high frequency (900 or 1200 pulses/sec) and it is kept on continuously. It is important to stress that with this implant there is, unlike other vestibular implants [3], no modulation of the pulse rate or pulse amplitude in response to the movement of the patient. The ES is 
composed of a constant pulse train delivered by three electrodes of a modified cochlear implant electrode. Each pulse is biphasic ( 25 microseconds per phase ) and delivered at a frequency of 900 or 1200 pulses per second. Three electrodes are activated so the stimulus is simply a constant train of pulses which will activate vestibular afferents at a very high rate, presumably duplicating the constant barrage of action potentials which healthy patients receive.

6. Stimulation phase. The patient is then re-tested on repeated occasions in the postoperative phase (in some patients this phase has lasted years). On each testing occasion this is done first to ensure the electrode is working. Fibrosis or changes in impedance may make the electrode non-functional in which case vestibular and clinical tests would be rendered meaningless. Then on each occasion they have been tested on the battery of tests of peripheral vestibular function, postural stability, gait performance and acceptability. Their results on these tests were then compared to their preoperative measures and their performance in comparison to the electrode OFF condition and measures of patient satisfaction (usually relying on patient subjective reports, or oscillopsia).

\section{Test Results.}

See Ramos Macias et al [1] for detailed results. On a priori grounds, we did not expect some vestibular tests to show changes: for example, we did not expect ES of saccular afferents to affect semicircular canal function (as measured by VOR gain using the video Head Impulse Test (vHIT)). However, it was possible that measures of otolith function could change as a result of the ES stimulation. We were particularly concerned to identify if there were improvements in tests of postural stability and gait performance.

\section{The Saccular Substitution Hypothesis}

With this saccular ES patients show improved postural stability and gait performance (e.g., measured by the DGI or CDP) and so the question of central importance is how could constant electrical stimulation of saccular afferents in these BVD patients cause this improved performance? We consider that this maintained stimulation is generating a barrage of action potentials in the saccular nerve which is substituting for the reduced or absent saccular afferent action potentials in these BVD patients. The saccular macula is unique in that saccular receptors are continuously stimulated by the force of gravity. There are a large number of primary saccular afferents (around 4000 in humans, [4,5]) and these saccular afferents have high resting discharge rates, (probably around 50-100 spikes/s in humans [6]) so each second a huge barrage of action potentials is continuously reaching the vestibular nuclei and the midline cerebellum, and we suggest, indirectly projecting on to other structures e.g. the basal ganglia. Bilateral vestibular loss will reduce or remove this sustained neural input and it appears that the high frequency electrical stimulation by the implanted vestibular electrode is substituting for this lost natural neural activation.

So, their loss is depriving their target nuclei of a huge amount of neural input which is presumably a major contributing factor to the poor performance of BVD patients on posture and gait tasks pre-operatively.

The questions then become:

1. Where do saccular afferents project to? and

2. How could their sustained activation influence posture and gait performance?

To address these questions, we present a brief overview of evidence about direct and indirect saccular projections and refer to the effect of damage to these target areas on patient performance. There is new evidence about how deep brain stimulation acts upon some of these target areas to improve patient gait and postural stability. In the following we consider the effects of ES of the basal ganglia, the cerebellum and the 
pedunculopontine nucleus (PPN) of the mesencephalic locomotor region in patients with deficits of motor control for improving postural stability and gait performance.

\section{Saccular Projections}

As is clear from the anatomical evidence of the direct and indirect central projections of saccular afferents, their neural activity has very widespread effects in motor control systems. Saccular afferents project to brainstem vestibular nuclei, to spinal cord, and to cerebellum, and indirectly through the cerebellum to the basal ganglia and to other structures which have been of great recent interest in motor control - especially the pedunculopontine nucleus (PPN) of the mesencephalic locomotor region (MLR). So, the activation of saccular afferents is indirectly activating many motor control systems.

Saccular afferents travel in the inferior vestibular nerve, along with afferents from the posterior semicircular canal. Saccular afferent fibres branch and send a thinner collateral branch to the cerebellum, with the thicker afferent branch projecting to the vestibular nuclei. Saccular fibres terminate in the lateral and inferior vestibular nuclei. Anatomical evidence shows that saccular afferents project both to vestibular nuclei and the cerebellum - specifically to the anterior midline structures including the uvula [7-9] and deep cerebellar nuclei [10]. see Figure 1.

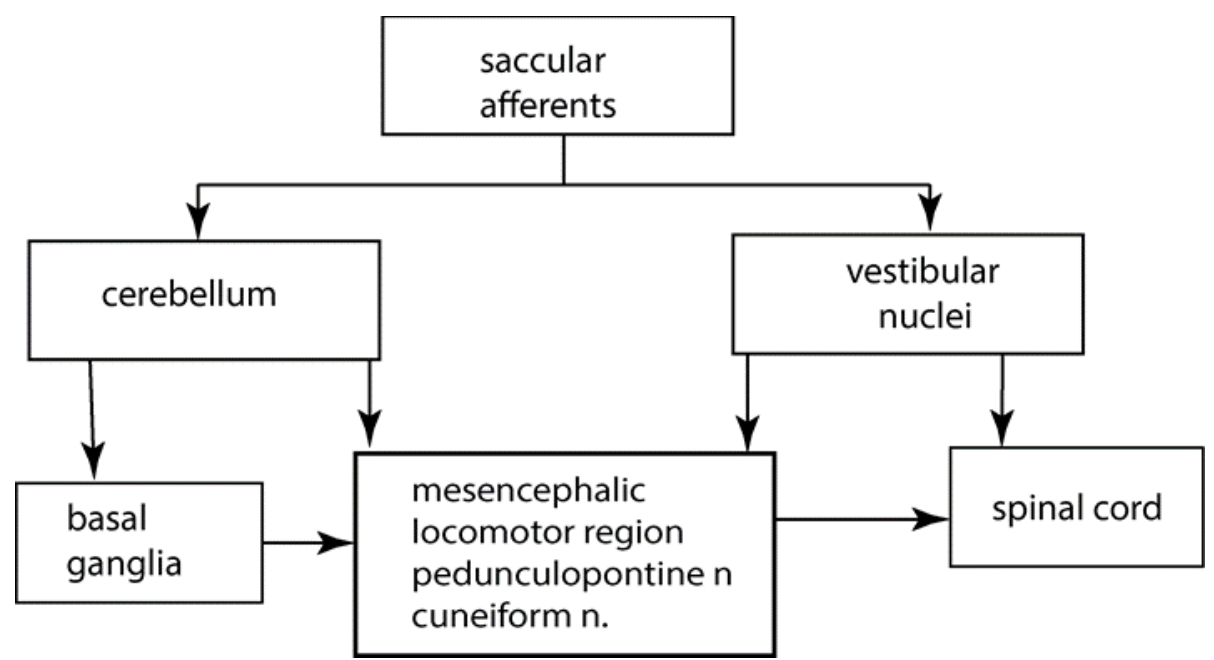

Figure 1. A greatly simplified schematic overview of some of the major structures involved in the control of gait and postural stability

\section{The role of the cerebellum in posture and gait.}

There is extensive evidence of the crucial role of the cerebellum in the control of balance and locomotion (see e.g. [11]). Cerebellar circuits connect with many brain and spinal cord nuclei. Cerebellar activity is required for motor behaviours ranging from coordination, to posture and balance and gait. This is clear from the deficits in these behaviours in human patients with localized cerebellar damage [11]. Projections from the deep cerebellar nuclei influence basal ganglia activity by afferents which project to the thalamic nuclei that project to the basal ganglia primarily the striatum [11]. Dijkstra reports the cerebellum is involved in postural control, as shown by image analysis [12]. Mori et al. [13] demonstrated in cats that stimulation of the midline cerebellar locomotor region, (the fastigial nucleus) can independently induce locomotion. Neuroimaging suggests a similar region exists in humans. In studies with mental imagery of gait or foot pedals, showed that active stepping during fMRI, causes focal increases in the fastigial nucleus of the cerebellum and cerebellar vermis [12]. The cuneiform nucleus also appears to be of interest.

All the studies suggested the cerebellum is implicated in all aspects of the pathophysiology of Parkinson's disease. One of the most characteristic signs of cerebellar damage 
is walking ataxia. Anterior cerebellar damage (to the so-called vestibulo cerebellum) leads to increased postural sway $[11,14,15]$. Cerebellar damage is also associated with hypermetric postural responses to surface displacements and impaired ability to learn responses to predictable perturbations, or step initiation [16]. Some of these deficits that are described for patients with cerebellar loss appear to be similar to those described for patients with bilateral vestibular loss.

It is not known exactly how the cerebellum normally contributes to walking, although recent work suggests that it plays a role in the generation of appropriate patterns of limb movements, dynamic regulation of balance and adaptation of posture and locomotion through practice $[17,18]$. Patients with cerebellar loss show excessive or diminished responses to perturbations, with poor control of equilibrium during motion and abnormal oscillation of the trunk. Gait ataxia is often described with distinctive features including variable foot placement, irregular foot trajectories, wide base of support, a veering path of movement, and abnormal inter-joint coordination [11]. These are similar to the gait disturbances of patients with BVD [1].

\section{Cerebellar locomotor region}

The cerebellar locomotor region is in the midline of the cerebellum [19] and as noted above, it is this region where saccular afferents terminate. Neurons from the central cerebellum project back to the vestibular nuclei $[4,7,8,10,20-23]$. Physiological studies from animals suggest that cerebellar control of posture, equilibrium, and locomotion are tightly controlled and localised in the medial zone. There are cerebellar projections to vestibular and reticular nuclei and also to the thalamus. Thus, the medial cerebellar zone can integrate spinal and vestibular inputs and influence motor pathways for walking.

\section{Cerebellum to Basal Ganglia}

Manto elaborates on the short latency connections between the cerebellum and the basal ganglia [24-26]. These connections may explain the cerebellar involvement in disorders commonly associated with basal ganglia dysfunction, or example, Parkinson's disease. Recently, cerebellar neurons are being stimulated in human patients by brain stimulation techniques, including transcranial magnetic stimulation, and transcranial direct current stimulation, to alleviate disturbances of motor control $[13,27-30][27,28]$. In an animal model, Miterko reports that neuromodulation of the cerebellum rescues movement in a mouse model of ataxia[27].

\section{The pedunculopontine nucleus (PPN)}

The mesencephalic locomotor region (MLR) consists of the pedunculopontine tegmental nucleus (PPN), the cuneiform nucleus, and the sub-cuneiform nucleus. It receives input from cerebellar nuclei and basal ganglia [31-33]. Imaging of the PPN during imagined walking shows that it is involved in control of postural stability [34-36] and electrical stimulation of PPN is a target for treating locomotion deficits [37] .

\section{Vestibular Nuclei to Basal Ganglia}

The most convincing evidence that there may be disynaptic projections from the vestibular nuclei to the basal ganglia was published by Lai et al. [38]. Using neuronal tracers, they reported that projections from the medial vestibular nucleus to the parafascicular nucleus (PFN) of the thalamus, synapse on neurons which project to the dorsolateral putamen of the striatum. This anatomical evidence suggested the possibility of a disynaptic pathway between the vestibular nuclei and the striatum, albeit from the medial vestibular nucleus rather than the inferior and lateral vestibular nuclei, where saccular afferents are known to terminate. The PFN is also strongly connected to the PPN [39]. Although there have been a small number of electrophysiological studies investigating whether electrical stimulation of the peripheral vestibular system can evoke field potentials and single unit activity in the striatum (see [39] and [40] for reviews), none of these can exclude the 
possibility that any responses arise via the cerebellum and none of them involved selective saccular stimulation. Nonetheless, there are many regions of the striatum which have not been explored, such the striatal tail, which is known to receive substantial visual and auditory sensory input and may also receive vestibular input (see [41] for a review). Whether saccular information is transmitted to this multisensory integration centre remains to be determined, but seems very likely.

\section{$\underline{\text { Gait }}$}

Normal gait is a complex process that involves concomitant balance and locomotion processes. A hierarchy of supraspinal regions send signals to the central pattern generators (CPGs) of the spinal cord [36,42]. Supraspinal regions modify stereotyped locomotion in certain situations such as initiating gait, turning, stopping, and avoiding obstacles. The locomotor network involves CPG's, mesencephalic locomotor region, the cerebellar locomotor areas, subthalamic locomotor region, and various cortical areas including frontal and parietal supplementary motor and motor areas [36].

\section{Contribution of the Otoliths to Spatial Awareness}

There has been increasing evidence that the otoliths, independently of the semi-circular canals, may be important for spatial orientation and awareness ('spatial cognition'). Although the effects of inferior vestibular nerve ES on spatial cognition have not been investigated quantitatively, it is conceivable that a part of the perceived improvement in function reported by patients receiving ES is due to its cognitive effects. Logically, due to its role in the perception of gravity, the saccule might be expected to be particularly important in providing a gravitational reference frame for other sensory information. It is difficult to surgically manipulate the saccule in animal models without affecting the other vestibular sensors. However, a recent study in rats reported that selective electrical stimulation of the saccule resulted in widespread activation of the bilateral hippocampus, with field potential amplitudes greater than for similar stimulation of the semi-circular canals [43]. An important source of information about the impact of having no otolithic function are the mutant mouse strains in which otoconia do not develop (e.g., "tilted Het" and "Otop" mice; see 40 for a review). Inevitably, the mice have neither saccular nor utricular function, so it is impossible to separate the two otolithic receptor systems; however, they have normal semicircular canal function. Quantitative gait analysis has not been performed on these otolith-deficient mouse models as yet; however, they do exhibit substantial deficits on the rotarod, in exploration and in performance in Y maze, radial arm maze, elevated plus maze and place recognition tasks (see 40 for a review). These results suggest that loss of otolithic function results in deficits in spatial cognition. Studies of the first 10 days of development indicate that Het mice develop abnormally, exhibiting abnormal responses in the righting reflex, cliff drop aversion and negative geotaxis tests [44]. There is also evidence that thalamic head direction cells and hippocampal place cells function abnormally in otolith-deficient mice (see 40 for a review). Taken together, these studies suggest that the otoliths are important for spatial awareness and orientation and that saccular ES may have a beneficial effect in BVD patients.

\section{Conclusion}

This review has shown the widespread direct and indirect projection of saccular afferent neural activity to brain structures controlling postural stability and gait performance. In patients with BVD this neural activity will be reduced or absent and we suggest that the constant ES is activating primary saccular afferents and so is substituting for the absent saccular afferent neural activity and so is acting to improve postural stability and gait.

Author Contributions: Conceptualization, IC, writing IC and PS, ARM, project administration ARM. All authors have read and agreed to the published version of the manuscript 
Funding: "This research received no external funding".

Institutional Review Board Statement: Not applicable

Informed Consent Statement: Not applicable

Data Availability Statement: Not applicable

Acknowledgments: We thank Kim Hawkins, Daniel Quinlan and Laura Fröhlich for their very helpful comments.

Conflicts of Interest: The authors declare no conflicts of interest.

\section{References}

1. Ramos Macias, A.; Ramos de Miguel, A.; Montesdeoca, I.R.; Barreiro, S.B.; C., F.G.J. Chronic electrical stimulation of the otolith organ: preliminary results in humans with bilateral vestibulopathy and sensorineural hearing loss. Audiology and Neurotology 2019, DOI: 10.1159/000503600, 1-12, doi:DOI: 10.1159/000503600.

2. Strupp, M.; Kim, J.S.; Murofushi, T.; Straumann, D.; Jen, J.C.; Rosengren, S.M.; Della Santina, C.C.; Kingma, H. Bilateral vestibulopathy: Diagnostic criteria Consensus document of the Classification Committee of the Barany Society. Journal of Vestibular Research-Equilibrium \& Orientation 2017, 27, 177-189, doi:10.3233/ves-170619.

3. Della Santina, C.C.; Migliaccio, A.A.; Patel, A.H. A multichannel semicircular canal neural prosthesis using electrical stimulation to restore 3-D vestibular sensation. IEEE Trans. Biomed. Eng. 2007, 54, 1016-1030, doi:10.1109/tbme.2007.894629.

4. Buttner-Ennever, J.A. A review of otolith pathways to brainstem and cerebellum. In Otolith Function in Spatial Orientation and Movement, Cohen, B., Hess, B.J.M., Eds. 1999; Vol. 871, pp. 51-64.

5. Won, S.L.; Suarez, C.; Honrubia, V.; Gomez, J. Morphological aspects of the human vestibular nerve. Laryngoscope 1990, 100, 756-764.

6. Goldberg, J.M. Afferent diversity and the organization of central vestibular pathways. Exp. Brain Res. 2000, 130, 277-297.

7. Maklad, A.; Fritzsch, B. Partial segregation of posterior crista and saccular fibers to the nodulus and uvula of the cerebellum in mice, and its development. Developmental Brain Research 2003, 140, 223-236, doi:10.1016/s01653806(02)00609-0.

8. Maklad, A.; Kamel, S.; Wong, E.; Fritzsch, B. Development and organization of polarity-specific segregation of primary vestibular afferent fibers in mice. Cell Tissue Res. 2010, 340, 303-321, doi:10.1007/s00441-010-0944-1.

9. Gacek, R.R. The course and central termination of first order neurons supplying vestibular endorgans in the cat. Acta Otolaryngol Suppl 1969, 254, 1-66.

10. Barmack, N.H.; Baughman, R.W.; Errico, P.; Shojaku, H. Vestibular primary afferent projection to the cerebellum of the rabbit. J. Comp. Neurol. 1993, 327, 521-534, doi:10.1002/cne.903270405.

11. Morton, S.M.; Bastian, A.J. Cerebellar control of balance and locomotion. Neuroscientist 2004, 10, 247-259, doi:10.1177/1073858404263517.

12. Dijkstra, B.W.; Bekkers, E.M.J.; Gilat, M.; de Rond, V.; Hardwick, R.M.; Nieuwboer, A. Functional neuroimaging of human postural control: A systematic review with meta-analysis. Neurosci. Biobehav. Rev. 2020, 115, 351-362,

doi:10.1016/j.neubiorev.2020.04.028. 
13. Mori, S.; Matsui, T.; Kuze, B.; Asanome, M.; Nakajima, K.; Matsuyama, K. Stimulation of a restricted region in the midline cerebellar white matter evokes coordinated quadrupedal locomotion in the decerebrate cat. J. Neurophysiol. 1999, 82, 290-300, doi:10.1152/jn.1999.82.1.290.

14. Mauritz, K.H.; Dichgans, J.; Hufschmidt, A. Quantitative-analysis of stance in late cortical cerebellar atrophy of the anterior lobe and other forms of cerebellar-ataxia. Brain 1979, 102, 461-482, doi:10.1093/brain/102.3.461.

15. Horak, F.B.; Diener, H.C. Cerebellar control of postural scaling and central set in stance. J. Neurophysiol. 1994, 72, 479-493, doi:10.1152/jn.1994.72.2.479.

16. Timmann, D.; Horak, F.B. Perturbed step initiation in cerebellar subjects: 2. Modification of anticipatory postural adjustments. Exp. Brain Res. 2001, 141, 110-120, doi:10.1007/s002210100858.

17. Bent, L.R.; Inglis, J.T.; McFadyen, B.J. Vestibular contributions across the execution of a voluntary forward step. Exp. Brain Res. 2002, 143, 100-105, doi:10.1007/s00221-001-0967-7.

18. Bent, L.R.; McFadyen, B.J.; Inglis, J.T. Vestibular contributions during human locomotor tasks. Exerc. Sport Sci. Rev. 2005, 33, 107-113, doi:10.1097/00003677-200507000-00002.

19. Jahn, K.; Deutschlader, A.; Stephan, T.; Kalla, R.; Wiesmann, M.; Strupp, M.; Brandt, T. Imaging human supraspinal locomotor centers in brainstem and cerebellum. Neuroimage 2008, 39, 786-792, doi:10.1016/j.neuroimage.2007.09.047.

20. Imagawa, M.; Graf, W.; Sato, H.; Suwa, H.; Isu, N.; Izumi, R.; Uchino, Y. Morphology of single afferents of the saccular macula in cats. Neurosci. Lett. 1998, 240, 127-130, doi:10.1016/s0304-3940(97)00944-0.

21. Korte, G.E.; Mugnaini, E. Cerebellar projection of the vestibular nerve in the cat. J. Comp. Neurol. 1979, 184, 265277, doi:10.1002/cne.901840204.

22. Uchino, Y.; Sato, H.; Suwa, H. Excitatory and inhibitory inputs from saccular afferents to single vestibular neurons in the cat. J. Neurophysiol. 1997, 78, 2186-2192.

23. Gacek, R.R. Course and central termination of 1 order neurons supplying vestibular endorgans in cat. Acta Otolaryngol. (Stockh.) 1969, S, 1-\&.

24. Bostan, A.C.; Dum, R.P.; Strick, P.L. The basal ganglia communicate with the cerebellum. Proc. Natl. Acad. Sci. U. S. A. 2010, 107, 8452-8456, doi:10.1073/pnas.1000496107.

25. Bostan, A.C.; Dum, R.P.; Strick, P.L. Cerebellar networks with the cerebral cortex and basal ganglia. Trends in Cognitive Sciences 2013, 17, 241-254, doi:10.1016/j.tics.2013.03.003.

26. Manto, M.; Argyropoulos, G.P.D.; Bocci, T.; Celnik, P.A.; Corben, L.A.; Guidetti, M.; Koch, G.; Priori, A.; Rothwell, J.C.; Sadnicka, A., et al. Consensus Paper: Novel Directions and Next Steps of Non-invasive Brain Stimulation of the Cerebellum in Health and Disease. Cerebellum 2021, 10.1007/s12311-021-01344-6, doi:10.1007/s12311-021-01344-6.

27. Miterko, L.N.; Baker, K.B.; Beckinghausen, J.; Bradnam, L.V.; Cheng, M.Y.; Cooperrider, J.; DeLong, M.R.; Gornati, S.V.; Hallett, M.; Heck, D.H., et al. Consensus Paper: Experimental Neurostimulation of the Cerebellum. Cerebellum 2019, 18, 1064-1097, doi:10.1007/s12311-019-01041-5.

28. Miterko, L.N.; Lin, T.; Zhou, J.; van der Heijden, M.E.; Beckinghausen, J.; White, J.J.; Sillitoe, R.V. Neuromodulation of the cerebellum rescues movement in a mouse model of ataxia. Nat. Commun. 2021, 12, doi:10.1038/s41467-02121417-8.

29. Ponce, G.V.; Klaus, J.; Schutter, D. A Brief History of Cerebellar Neurostimulation. Cerebellum 2021, 10.1007/s12311-021-01310-2, doi:10.1007/s12311-021-01310-2. 
30. Workman, C.D.; Fietsam, A.C.; Uc, E.Y.; Rudroff, T. Cerebellar Transcranial Direct Current Stimulation in People with Parkinson's Disease: A Pilot Study. Brain Sciences 2020, 10, doi:10.3390/brainsci10020096.

31. Hazrati, L.N.; Parent, A. Projection from the deep cerebellar nuclei to the pedunculopontine nucleus in the squirrel-monkey. Brain Res. 1992, 585, 267-271, doi:10.1016/0006-8993(92)91216-2.

32. Raghu, A.L.B.; Parker, T.; Zand, A.P.D.; Payne, S.; Andersson, J.; Stein, J.; Aziz, T.Z.; Green, A.L. Tractography patterns of pedunculopontine nucleus deep brain stimulation. J. Neural Transm. 2021, 128, 659-670, doi:10.1007/s00702021-02327-x.

33. Tubert, C.; Galtieri, D.; Surmeier, D.J. The pedunclopontine nucleus and Parkinson's disease. Neurobiol. Dis. 2019, 128, 3-8, doi:10.1016/j.nbd.2018.08.017.

34. Takakusaki, K.; Chiba, R.; Nozu, T.; Okumura, T. Brainstem control of locomotion and muscle tone with special reference to the role of the mesopontine tegmentum and medullary reticulospinal systems. J. Neural Transm. 2016, 123, 695-729, doi:10.1007/s00702-015-1475-4.

35. Molina, R.; Hass, C.J.; Sowalsky, K.; Schmitt, A.C.; Opri, E.; Roper, J.A.; Martinez-Ramirez, D.; Hess, C.W.; Foote, K.D.; Okun, M.S., et al. Neurophysiological Correlates of Gait in the Human Basal Ganglia and the PPN Region in Parkinson's Disease. Frontiers in Human Neuroscience 2020, 14, doi:10.3389/fnhum.2020.00194.

36. Takakusaki, K. Functional Neuroanatomy for Posture and Gait Control. Journal of Movement Disorders 2017, 10, 117, doi:10.14802/jmd.16062.

37. Noga, B.R.; Guest, J.D. Combined neuromodulatory approaches in the central nervous system for treatment of spinal cord injury. Curr. Opin. Neurol. 2021, 34, 804-811, doi:10.1097/wco.0000000000000999.

38. Lai, H.; Tsumori, T.; Shiroyama, T.; Yokota, S.; Nakano, K.; Yasui, Y. Morphological evidence for a vestibulothalamo-striatal pathway via the parafascicular nucleus in the rat. Brain Res. 2000, 872, 208-214, doi:10.1016/s00068993(00)02457-4.

39. Stiles, L.; Smith, P.F. The vestibular-basal ganglia connection: Balancing motor control. Brain Res. 2015, 1597, 180188, doi:10.1016/j.brainres.2014.11.063.

40. Smith, P.F. The Growing Evidence for the Importance of the Otoliths in Spatial Memory. Frontiers in Neural Circuits 2019, 13, doi:10.3389/fncir.2019.00066.

41. Valjent, E.; Gangarossa, G. The Tail of the Striatum: From Anatomy to Connectivity and Function. Trends Neurosci. 2021, 44, 203-214, doi:10.1016/j.tins.2020.10.016.

42. Cai, J.Y.; Lee, S.; Ba, F.; Garg, S.; Kim, L.J.; Liu, A.P.; Kim, D.; Wang, Z.J.; McKeown, M.J. Galvanic Vestibular Stimulation (GVS) Augments Deficient Pedunculopontine Nucleus (PPN) Connectivity in Mild Parkinson's Disease: fMRI Effects of Different Stimuli. Front. Neurosci. 2018, 12, doi:10.3389/hin3.2018.00101.

43. Hitier, M.; Zhang, Y.F.; Sato, G.; Besnard, S.; Zheng, Y.W.; Smith, P.F. Stratification of hippocampal electrophysiological activation evoked by selective electrical stimulation of different angular and linear acceleration sensors in the rat peripheral vestibular system. Hear. Res. 2021, 403, doi:10.1016/j.heares.2021.108173.

44. Le Gall, A.; Hilber, P.; Chesneau, C.; Bulla, J.; Toulouse, J.; Machado, M.L.; Philoxene, B.; Smith, P.F.; Besnard, S. The critical role of vestibular graviception during cognitive-motor development. Behav. Brain Res. 2019, 372, doi:10.1016/j.bbr.2019.112040. 